Acta Crystallographica Section E

Structure Reports

Online

ISSN 1600-5368

\section{9,10-Bis\{2-[1-(2-pyridylmethyl)- imidazolium-3-yl]ethoxy\}anthracene bis(hexafluoridophosphate)}

\section{Li-Hui Guo, Da-Bin Qin,* Shao-Jin Gu, Gui-Yu Wang and Jie-Wei Luo}

School of Chemistry and Chemical Engineering, China West Normal University, Nanchong 637002, People's Republic of China

Correspondence e-mail: qindabincwnu@yahoo.com.cn

Received 13 November 2007; accepted 27 November 2007

Key indicators: single-crystal X-ray study; $T=294 \mathrm{~K}$; mean $\sigma(\mathrm{C}-\mathrm{C})=0.007 \AA$; disorder in solvent or counterion; $R$ factor $=0.055 ; w R$ factor $=0.159$; data-toparameter ratio $=8.9$.

The cation of the title compound, $\mathrm{C}_{36} \mathrm{H}_{34} \mathrm{~N}_{6} \mathrm{O}_{2}{ }^{2+} \cdot 2 \mathrm{PF}_{6}{ }^{-}$, lies across a crystallographic inversion centre. The imidazole and pyridine rings form dihedral angles of $82.28(5)^{\circ}$ and $11.87(7)^{\circ}$, respectively, with the anthracene ring system. The crystal packing is stabilized by $\pi-\pi$ interactions between the pyridine ring and the central ring of anthracene, with a ring centroid-centroid distance of 3.684 (3) $\AA$. The $\mathrm{PF}_{6}{ }^{-}$anion is disordered over three different positions with occupancies of $0.284(6), 0.354(8)$ and $0.362(9)$.

\section{Related literature}

For the synthesis, see: Liu et al. (2003). For related structures, see: Liu et al. (2007); Pei et al. (2005); Qin et al. (2006).

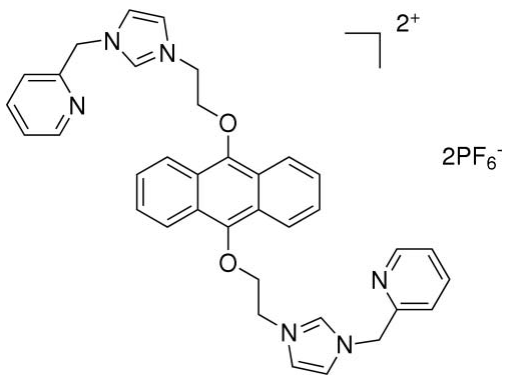

\section{Experimental}

Crystal data

$\begin{array}{ll}\mathrm{C}_{36} \mathrm{H}_{34} \mathrm{~N}_{6} \mathrm{O}_{2}{ }^{2+} \cdot 2 \mathrm{PF}_{6}{ }^{-} & \gamma=65.598(7)^{\circ} \\ M_{r}=872.63 & V=943.2(7) \AA^{3} \\ \text { Triclinic, } P \overline{1} & Z=1 \\ a=8.829(4) \AA & \text { Mo } K \alpha \text { radiation } \\ b=9.811(4) \AA & \mu=0.22 \mathrm{~mm}^{-1} \\ c=12.586(5) \AA & T=294(2) \mathrm{K} \\ \alpha=72.382(7)^{\circ} & 0.24 \times 0.20 \times 0.18 \mathrm{~mm}\end{array}$

$\beta=78.759(7)^{\circ}$

Data collection

Bruker SMART CCD area-detector diffractometer

Absorption correction: multi-scan (SADABS; Sheldrick, 1996)

$T_{\min }=0.950, T_{\max }=0.962$

Refinement

$R\left[F^{2}>2 \sigma\left(F^{2}\right)\right]=0.055$

$w R\left(F^{2}\right)=0.159$

$S=1.01$

3307 reflections

373 parameters

319 restraints

$\mathrm{H}$-atom parameters constrained

$\Delta \rho_{\text {max }}=0.33 \mathrm{e}^{-3}$

$\Delta \rho_{\min }=-0.24 \mathrm{e}^{-3}$
Data collection: SMART (Bruker, 1998); cell refinement: SAINT (Bruker, 1999); data reduction: $S A I N T$; $\operatorname{program}(\mathrm{s})$ used to solve structure: SHELXS97 (Sheldrick, 1997); program(s) used to refine structure: SHELXL97 (Sheldrick, 1997); molecular graphics: SHELXTL (Bruker, 1999); software used to prepare material for publication: SHELXTL.

The authors thank the Scientific Research Fund Projects of China West Normal University (grant No. 06B003) and the Youth Fund Projects of Sichuan Educational Department (grant No. 2006B039).

Supplementary data and figures for this paper are available from the IUCr electronic archives (Reference: CI2523).

\section{References}

Bruker (1998). SMART. Bruker AXS Inc., Madison, Wisconsin, USA. Bruker (1999). SAINT and SHELXTL. Bruker AXS Inc., Madison, Wisconsin, USA.

Liu, B., Chen, W. Z. \& Jin, S. W. (2007). Organometallics, 26, 3660-3667.

Liu, Q. X., Xu, F. B., Li, Q. S., Zeng, X. S., Leng, X. B., Chou, Y. L. \& Zhang, Z. Z. (2003). Organometallics, 22, 309-314.

Pei, L. C., Chun, L. L., Chiu, F. C., Ching, H. H. \& Hon, M. L. (2005). Organometallics, 24, 6169-6178.

Qin, D. B., Xu, F. B., Wan, X. J., Zhao, Y. J. \& Zhang, Z. Z. (2006). Tetrahedron Lett. 47, 5641-5643.

Sheldrick, G. M. (1996). SADABS. University of Göttingen, Germany.

Sheldrick, G. M. (1997). SHELXS97 and SHELXL97. University of Göttingen, Germany. 


\section{supporting information}

Acta Cryst. (2008). E64, o189 [https://doi.org/10.1107/S1600536807063842]

\section{9,10-Bis\{2-[1-(2-pyridylmethyl) imidazolium-3-yl] ethoxy\}anthracene bis-}

\section{(hexafluoridophosphate)}

\section{Li-Hui Guo, Da-Bin Qin, Shao-Jin Gu, Gui-Yu Wang and Jie-Wei Luo}

\section{S1. Comment}

Imidazolium salts or its derivatives or analogs are used as ionic liquids, and as catalysts in many organic transformation processes. They are also used to obtain N-heterocyclic carbene complexes. We report here the crystal structure of the title compound.

The asymmetric unit of the title compound contains one-half of the organic cation and a $\mathrm{PF}_{6}$ counter ion. The cation lies on a crystallographic inversion center. Bond lengths and angles in the title molecule (Fig. 1) are within normal ranges. The imidazole ring is almost perpendicular to the anthracene ring system, with a dihedral angle of $82.28(5)^{\circ}$, and the pyridine ring is almost parallel to the anthracene ring system, with a dihedral angle of $11.87(7)^{\circ}$. The crystal packing is stabilized by $\pi$ - $\pi$ interactions between pyridine ring and central ring of anthracene [centroid-centroid distance is 3.684 (3) $\%$ A.

\section{S2. Experimental}

The title compound was prepared according to the reported procedure of Liu et al. (2003). Yellow single crystals suitable for X-ray diffraction were obtained by recrystallization from acetonitrile.

\section{S3. Refinement}

The $\mathrm{PF}_{6}$ group is disordered over three different positions with refined occupancies of $0.284(6), 0.354$ (8) and 0.362 (9). The $\mathrm{P}-\mathrm{F}$ distances were restrained to 1.56 (1) $\AA$, and the displacement parameters of disordered $\mathrm{F}$ atoms were restrained to an approximate isotropic behaviour, and also restrained to have the same $\mathrm{U}^{\mathrm{ij}}$ components. $\mathrm{H}$ atoms were placed in calculated positions with $\mathrm{C}-\mathrm{H}=0.93-0.97 \AA$, and refined using a riding model approximation, with $U_{\text {iso }}(\mathrm{H})=1.2_{\mathrm{eq}}(\mathrm{C})$. 


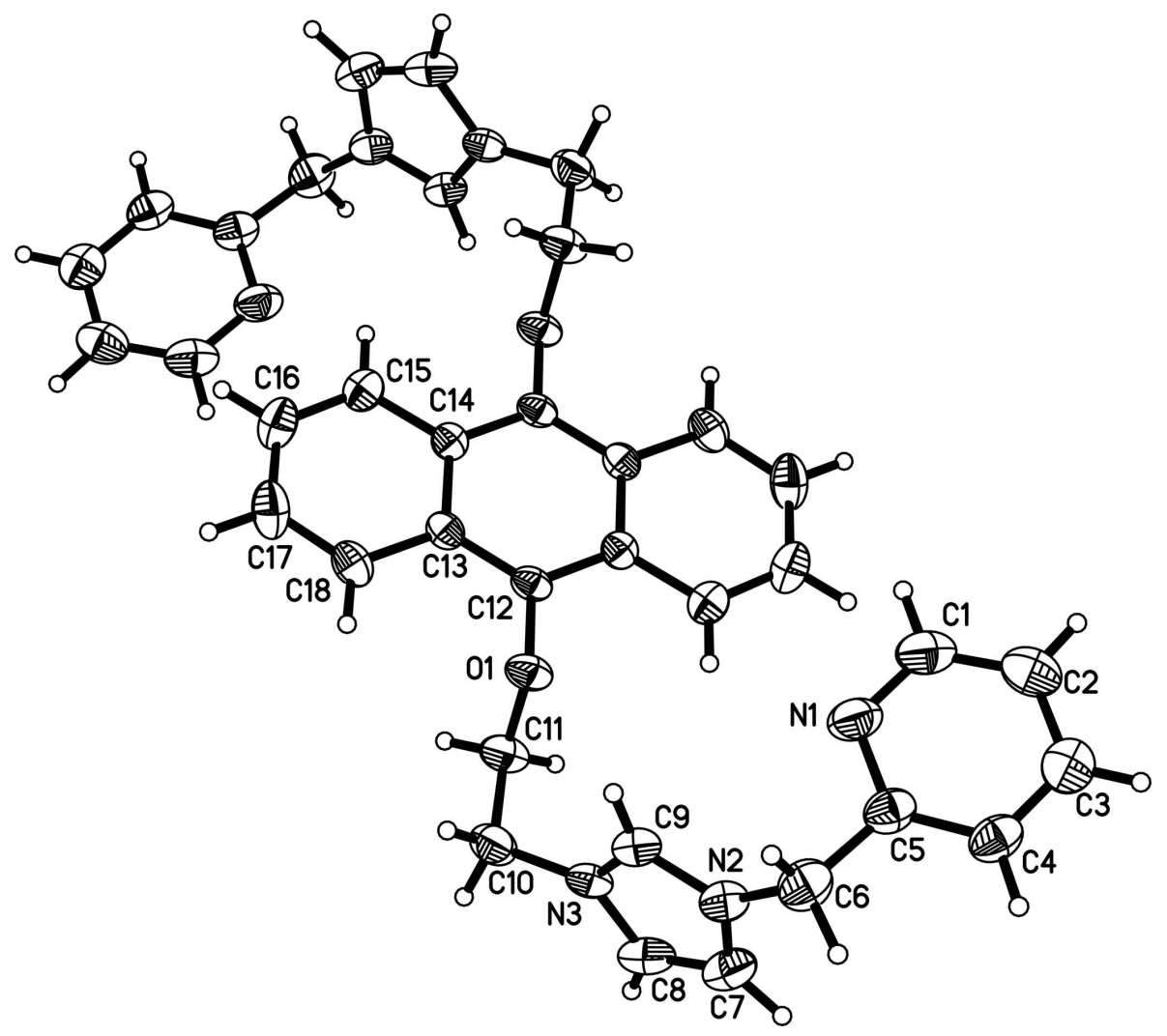

Figure 1

View of the cationic unit in the title compound. Displacement ellipsoids are drawn at the $30 \%$ probability level.

Unlabelled atoms are related to labelled atoms by the symmetry operation $(1-x, 1-y, 2-z)$.

9,10-Bis\{2-[1-(2-pyridylmethyl)imidazolium-3-yl] ethoxy\}anthracene bis(hexafluoridophosphate)

Crystal data

$\mathrm{C}_{36} \mathrm{H}_{34} \mathrm{~N}_{6} \mathrm{O}_{2}{ }^{2+} \cdot 2 \mathrm{PF}_{6}$

$M_{r}=872.63$

Triclinic, $P \overline{1}$

Hall symbol: -P 1

$a=8.829(4) \AA$

$b=9.811(4) \AA$

$c=12.586(5) \AA$

$\alpha=72.382(7)^{\circ}$

$\beta=78.759(7)^{\circ}$

$\gamma=65.598(7)^{\circ}$

$V=943.2(7) \AA^{3}$

Data collection

Bruker SMART CCD area-detector diffractometer

Radiation source: fine-focus sealed tube

Graphite monochromator

$\varphi$ and $\omega$ scans

Absorption correction: multi-scan

(SADABS; Sheldrick, 1996)

$T_{\min }=0.950, T_{\max }=0.962$
$Z=1$

$F(000)=446$

$D_{\mathrm{x}}=1.536 \mathrm{Mg} \mathrm{m}^{-3}$

Mo $K \alpha$ radiation, $\lambda=0.71073 \AA$

Cell parameters from 1209 reflections

$\theta=2.7-21.5^{\circ}$

$\mu=0.22 \mathrm{~mm}^{-1}$

$T=294 \mathrm{~K}$

Block, yellow

$0.24 \times 0.20 \times 0.18 \mathrm{~mm}$

4911 measured reflections

3307 independent reflections

1792 reflections with $I>2 \sigma(I)$

$R_{\text {int }}=0.027$

$\theta_{\text {max }}=25.0^{\circ}, \theta_{\text {min }}=1.7^{\circ}$

$h=-10 \rightarrow 10$

$k=-11 \rightarrow 6$

$l=-14 \rightarrow 14$ 


\section{Refinement}

Refinement on $F^{2}$

Least-squares matrix: full

$R\left[F^{2}>2 \sigma\left(F^{2}\right)\right]=0.055$

$w R\left(F^{2}\right)=0.159$

$S=1.01$

3307 reflections

373 parameters

319 restraints

Primary atom site location: structure-invariant direct methods
Secondary atom site location: difference Fourier map

Hydrogen site location: inferred from neighbouring sites

$\mathrm{H}$-atom parameters constrained

$w=1 /\left[\sigma^{2}\left(F_{\mathrm{o}}^{2}\right)+(0.0713 P)^{2}+0.3109 P\right]$ where $P=\left(F_{\mathrm{o}}^{2}+2 F_{\mathrm{c}}^{2}\right) / 3$

$(\Delta / \sigma)_{\max }=0.015$

$\Delta \rho_{\max }=0.33 \mathrm{e}^{-3}$

$\Delta \rho_{\min }=-0.24$ e $\AA^{-3}$

Special details

Geometry. All e.s.d.'s (except the e.s.d. in the dihedral angle between two l.s. planes) are estimated using the full covariance matrix. The cell e.s.d.'s are taken into account individually in the estimation of e.s.d.'s in distances, angles and torsion angles; correlations between e.s.d.'s in cell parameters are only used when they are defined by crystal symmetry. An approximate (isotropic) treatment of cell e.s.d.'s is used for estimating e.s.d.'s involving 1.s. planes.

Refinement. Refinement of $F^{2}$ against ALL reflections. The weighted $R$-factor $w R$ and goodness of fit $S$ are based on $F^{2}$, conventional $R$-factors $R$ are based on $F$, with $F$ set to zero for negative $F^{2}$. The threshold expression of $F^{2}>\sigma\left(F^{2}\right)$ is used only for calculating $R$-factors(gt) etc. and is not relevant to the choice of reflections for refinement. $R$-factors based on $F^{2}$ are statistically about twice as large as those based on $F$, and $R$ - factors based on ALL data will be even larger.

Fractional atomic coordinates and isotropic or equivalent isotropic displacement parameters $\left(\AA^{2}\right)$

\begin{tabular}{|c|c|c|c|c|c|}
\hline & $x$ & $y$ & $z$ & $U_{\text {iso }} * / U_{\text {eq }}$ & Occ. $(<1)$ \\
\hline $\mathrm{O} 1$ & $0.3505(3)$ & $0.6951(3)$ & 0.80589 (19) & $0.0542(7)$ & \\
\hline N1 & $-0.1667(4)$ & 1.0465 (4) & $0.9161(3)$ & $0.0759(11)$ & \\
\hline $\mathrm{N} 2$ & $-0.1181(4)$ & 1.0404 (4) & $0.6845(3)$ & $0.0594(9)$ & \\
\hline N3 & $0.0945(4)$ & $0.8468(4)$ & $0.6444(2)$ & $0.0537(9)$ & \\
\hline $\mathrm{C} 1$ & $-0.2180(7)$ & $1.0165(6)$ & $1.0243(4)$ & $0.0824(14)$ & \\
\hline H1 & -0.1385 & 0.9512 & 1.0749 & $0.099 *$ & \\
\hline $\mathrm{C} 2$ & $-0.3809(7)$ & $1.0760(6)$ & $1.0655(4)$ & $0.0786(14)$ & \\
\hline $\mathrm{H} 2$ & -0.4105 & 1.0523 & 1.1420 & $0.094 *$ & \\
\hline $\mathrm{C} 3$ & $-0.4988(6)$ & $1.1708(5)$ & $0.9918(5)$ & $0.0741(13)$ & \\
\hline H3 & -0.6107 & 1.2126 & 1.0168 & $0.089 *$ & \\
\hline $\mathrm{C} 4$ & $-0.4483(5)$ & $1.2030(5)$ & $0.8795(4)$ & $0.0665(12)$ & \\
\hline $\mathrm{H} 4$ & -0.5260 & 1.2661 & 0.8273 & $0.080^{*}$ & \\
\hline $\mathrm{C} 5$ & $-0.2824(5)$ & $1.1410(5)$ & $0.8457(4)$ & $0.0623(11)$ & \\
\hline C6 & $-0.2179(5)$ & $1.1793(5)$ & $0.7253(4)$ & $0.0747(13)$ & \\
\hline H6A & -0.3115 & 1.2428 & 0.6799 & $0.090^{*}$ & \\
\hline H6B & -0.1501 & 1.2389 & 0.7172 & $0.090^{*}$ & \\
\hline $\mathrm{C} 7$ & $-0.1765(6)$ & $0.9587(6)$ & $0.6444(4)$ & $0.0714(13)$ & \\
\hline $\mathrm{H} 7$ & -0.2876 & 0.9820 & 0.6362 & $0.086^{*}$ & \\
\hline $\mathrm{C} 8$ & $-0.0454(6)$ & $0.8389(6)$ & $0.6192(3)$ & $0.0690(13)$ & \\
\hline $\mathrm{H} 8$ & -0.0484 & 0.7637 & 0.5899 & $0.083 *$ & \\
\hline C9 & $0.0470(5)$ & $0.9692(5)$ & $0.6840(3)$ & $0.0571(11)$ & \\
\hline H9 & 0.1176 & 1.0009 & 0.7079 & $0.069 *$ & \\
\hline $\mathrm{C} 10$ & $0.2667(5)$ & $0.7415(5)$ & $0.6272(3)$ & $0.0697(13)$ & \\
\hline $\mathrm{H} 10 \mathrm{~A}$ & 0.3375 & 0.8007 & 0.6007 & $0.084^{*}$ & \\
\hline H10B & 0.2736 & 0.6916 & 0.5697 & $0.084 *$ & \\
\hline
\end{tabular}




\begin{tabular}{|c|c|c|c|c|c|}
\hline C11 & $0.3307(5)$ & $0.6199(5)$ & $0.7318(3)$ & $0.0616(11)$ & \\
\hline H11A & 0.2521 & 0.5702 & 0.7660 & $0.074 *$ & \\
\hline H11B & 0.4368 & 0.5418 & 0.7143 & $0.074 *$ & \\
\hline $\mathrm{C} 12$ & $0.4252(4)$ & $0.5939(4)$ & $0.9027(3)$ & $0.0440(9)$ & \\
\hline $\mathrm{C} 13$ & $0.5991(4)$ & $0.5322(4)$ & $0.9005(3)$ & $0.0449(9)$ & \\
\hline C14 & $0.6758(4)$ & $0.4338(4)$ & $1.0009(3)$ & $0.0438(9)$ & \\
\hline $\mathrm{C} 15$ & $0.8534(4)$ & $0.3686(5)$ & 0.9977 (4) & $0.0590(11)$ & \\
\hline H15 & 0.9055 & 0.3045 & 1.0623 & $0.071^{*}$ & \\
\hline $\mathrm{C} 16$ & $0.9462(5)$ & $0.3990(5)$ & $0.9021(4)$ & $0.0722(13)$ & \\
\hline H16 & 1.0620 & 0.3538 & 0.9010 & $0.087^{*}$ & \\
\hline $\mathrm{C} 17$ & $0.8715(5)$ & $0.4974(5)$ & $0.8043(4)$ & $0.0749(14)$ & \\
\hline H17 & 0.9382 & 0.5187 & 0.7396 & $0.090^{*}$ & \\
\hline $\mathrm{C} 18$ & $0.7031(5)$ & $0.5624(5)$ & $0.8023(3)$ & $0.0592(11)$ & \\
\hline H18 & 0.6555 & 0.6269 & 0.7363 & $0.071^{*}$ & \\
\hline P1 & $0.71210(13)$ & $0.71357(15)$ & $0.41845(9)$ & $0.0645(4)$ & \\
\hline F1 & $0.551(2)$ & $0.702(3)$ & $0.3935(19)$ & $0.114(6)$ & $0.284(6)$ \\
\hline $\mathrm{F} 2$ & $0.789(2)$ & $0.5628(17)$ & $0.3757(17)$ & $0.092(4)$ & $0.284(6)$ \\
\hline F3 & $0.758(2)$ & $0.811(2)$ & $0.3001(11)$ & $0.111(4)$ & $0.284(6)$ \\
\hline F4 & $0.6560(19)$ & $0.8681(17)$ & $0.4551(19)$ & $0.134(5)$ & $0.284(6)$ \\
\hline F5 & $0.662(2)$ & $0.615(2)$ & $0.5320(11)$ & $0.130(5)$ & $0.284(6)$ \\
\hline F6 & $0.8857(15)$ & $0.655(2)$ & $0.4618(15)$ & $0.115(5)$ & $0.284(6)$ \\
\hline F1A & $0.5497(16)$ & $0.672(2)$ & $0.4454(14)$ & $0.119(5)$ & $0.354(8)$ \\
\hline F2A & $0.7523(17)$ & $0.631(2)$ & $0.3229(13)$ & $0.093(4)$ & $0.354(8)$ \\
\hline F3A & $0.590(2)$ & $0.8701(16)$ & $0.3574(15)$ & $0.144(5)$ & $0.354(8)$ \\
\hline F4A & $0.6405(16)$ & $0.7923(18)$ & $0.5218(10)$ & $0.097(4)$ & $0.354(8)$ \\
\hline F5A & $0.761(2)$ & $0.5581(15)$ & $0.5135(13)$ & $0.130(4)$ & $0.354(8)$ \\
\hline F6A & $0.8703(15)$ & $0.7530(19)$ & $0.3841(15)$ & $0.114(5)$ & $0.354(8)$ \\
\hline F1B & $0.5218(10)$ & $0.776(2)$ & $0.4093(15)$ & $0.112(4)$ & $0.362(9)$ \\
\hline F2B & $0.7395(16)$ & $0.722(2)$ & $0.2880(7)$ & $0.101(4)$ & $0.362(9)$ \\
\hline F3B & $0.661(2)$ & $0.8926(11)$ & $0.3589(17)$ & $0.123(4)$ & $0.362(9)$ \\
\hline F4B & $0.6951(19)$ & $0.712(2)$ & $0.5411(7)$ & $0.108(4)$ & $0.362(9)$ \\
\hline F5B & $0.8139(15)$ & $0.5346(9)$ & $0.4402(17)$ & $0.109(4)$ & $0.362(9)$ \\
\hline F6B & $0.8931(11)$ & $0.7078(15)$ & $0.4201(10)$ & $0.066(3)$ & $0.362(9)$ \\
\hline
\end{tabular}

Atomic displacement parameters $\left(\AA^{2}\right)$

\begin{tabular}{lllllll}
\hline & $U^{11}$ & $U^{22}$ & $U^{33}$ & $U^{12}$ & $U^{13}$ & $U^{23}$ \\
\hline $\mathrm{O} 1$ & $0.0699(16)$ & $0.0451(16)$ & $0.0411(14)$ & $-0.0139(13)$ & $-0.0158(12)$ & $-0.0056(12)$ \\
$\mathrm{N} 1$ & $0.070(2)$ & $0.078(3)$ & $0.075(3)$ & $-0.015(2)$ & $-0.029(2)$ & $-0.016(2)$ \\
$\mathrm{N} 2$ & $0.062(2)$ & $0.056(2)$ & $0.057(2)$ & $-0.0193(19)$ & $-0.0174(16)$ & $-0.0072(18)$ \\
$\mathrm{N} 3$ & $0.066(2)$ & $0.055(2)$ & $0.0356(17)$ & $-0.0213(18)$ & $-0.0148(15)$ & $-0.0023(16)$ \\
$\mathrm{C} 1$ & $0.093(4)$ & $0.076(3)$ & $0.079(4)$ & $-0.022(3)$ & $-0.036(3)$ & $-0.016(3)$ \\
$\mathrm{C} 2$ & $0.112(4)$ & $0.070(3)$ & $0.071(3)$ & $-0.047(3)$ & $-0.011(3)$ & $-0.023(3)$ \\
$\mathrm{C} 3$ & $0.078(3)$ & $0.061(3)$ & $0.097(4)$ & $-0.032(3)$ & $-0.008(3)$ & $-0.032(3)$ \\
C4 & $0.064(3)$ & $0.051(3)$ & $0.086(3)$ & $-0.015(2)$ & $-0.024(2)$ & $-0.017(2)$ \\
C5 & $0.064(3)$ & $0.046(3)$ & $0.073(3)$ & $-0.013(2)$ & $-0.021(2)$ & $-0.012(2)$ \\
C6 & $0.071(3)$ & $0.058(3)$ & $0.084(3)$ & $-0.014(2)$ & $-0.019(2)$ & $-0.009(3)$ \\
C7 & $0.068(3)$ & $0.077(3)$ & $0.075(3)$ & $-0.032(3)$ & $-0.027(2)$ & $-0.006(3)$
\end{tabular}




$\begin{array}{lllllll}\text { C8 } & 0.090(3) & 0.068(3) & 0.060(3) & -0.038(3) & -0.032(2) & -0.004(2) \\ \text { C9 } & 0.061(3) & 0.059(3) & 0.053(2) & -0.022(2) & -0.0181(19) & -0.008(2) \\ \text { C10 } & 0.076(3) & 0.076(3) & 0.045(2) & -0.015(2) & -0.008(2) & -0.014(2) \\ \text { C11 } & 0.078(3) & 0.052(3) & 0.047(2) & -0.011(2) & -0.018(2) & -0.014(2) \\ \text { C12 } & 0.057(2) & 0.035(2) & 0.035(2) & -0.0121(18) & -0.0091(17) & -0.0064(17) \\ \text { C13 } & 0.053(2) & 0.042(2) & 0.040(2) & -0.0203(18) & -0.0008(17) & -0.0102(18) \\ \text { C14 } & 0.047(2) & 0.038(2) & 0.045(2) & -0.0141(17) & -0.0026(17) & -0.0126(18) \\ \text { C15 } & 0.051(2) & 0.058(3) & 0.065(3) & -0.017(2) & -0.007(2) & -0.015(2) \\ \text { C16 } & 0.046(2) & 0.070(3) & 0.090(4) & -0.015(2) & 0.001(2) & -0.018(3) \\ \text { C17 } & 0.065(3) & 0.068(3) & 0.077(3) & -0.027(2) & 0.022(2) & -0.012(3) \\ \text { C18 } & 0.070(3) & 0.058(3) & 0.045(2) & -0.029(2) & 0.006(2) & -0.007(2) \\ \text { P1 } & 0.0483(6) & 0.0874(10) & 0.0528(7) & -0.0162(6) & -0.0078(5) & -0.0219(7) \\ \text { F1 } & 0.084(7) & 0.149(10) & 0.128(9) & -0.048(6) & -0.055(6) & -0.025(8) \\ \text { F2 } & 0.114(7) & 0.086(7) & 0.091(8) & -0.042(5) & -0.007(6) & -0.036(6) \\ \text { F3 } & 0.134(7) & 0.097(7) & 0.092(7) & -0.060(6) & 0.002(6) & 0.003(6) \\ \text { F4 } & 0.158(7) & 0.100(7) & 0.110(7) & -0.007(6) & 0.006(7) & -0.051(6) \\ \text { F5 } & 0.126(7) & 0.137(7) & 0.084(6) & -0.032(6) & 0.017(6) & -0.008(6) \\ \text { F6 } & 0.098(6) & 0.126(8) & 0.114(8) & -0.037(6) & -0.050(6) & -0.003(6) \\ \text { F1A } & 0.070(5) & 0.154(8) & 0.126(8) & -0.060(5) & 0.011(5) & -0.010(7) \\ \text { F2A } & 0.109(6) & 0.085(7) & 0.090(7) & -0.026(6) & -0.001(5) & -0.048(6) \\ \text { F3A } & 0.118(8) & 0.121(7) & 0.146(7) & -0.023(6) & -0.030(7) & 0.010(6) \\ \text { F4A } & 0.116(6) & 0.101(7) & 0.050(5) & -0.015(5) & -0.001(5) & -0.026(5) \\ \text { F5A } & 0.128(7) & 0.110(6) & 0.099(6) & -0.020(5) & -0.011(6) & 0.011(5) \\ \text { F6A } & 0.106(6) & 0.113(8) & 0.127(9) & -0.072(6) & -0.002(6) & 0.005(6) \\ \text { F1B } & 0.051(4) & 0.129(8) & 0.128(7) & -0.016(5) & -0.009(4) & -0.019(7) \\ \text { F2B } & 0.122(6) & 0.108(8) & 0.069(5) & -0.030(6) & -0.003(4) & -0.037(5) \\ \text { F3B } & 0.108(7) & 0.093(6) & 0.132(7) & -0.032(5) & -0.023(6) & 0.022(6) \\ \text { F4B } & 0.142(7) & 0.131(8) & 0.047(4) & -0.040(6) & -0.019(4) & -0.024(5) \\ \text { F5B } & 0.126(6) & 0.077(5) & 0.113(7) & -0.029(4) & -0.015(6) & -0.020(5) \\ \text { F6B } & 0.061(4) & 0.075(7) & 0.060(6) & -0.040(4) & -0.023(4) & 0.019(5)\end{array}$

Geometric parameters $\left(\AA,{ }^{\circ}\right)$

\begin{tabular}{llll}
\hline $\mathrm{O} 1-\mathrm{C} 12$ & $1.395(4)$ & $\mathrm{C} 11-\mathrm{H} 11 \mathrm{~A}$ & 0.97 \\
$\mathrm{O} 1-\mathrm{C} 11$ & $1.425(4)$ & $\mathrm{C} 11-\mathrm{H} 11 \mathrm{~B}$ & 0.97 \\
$\mathrm{~N} 1-\mathrm{C} 5$ & $1.327(5)$ & $\mathrm{C} 12-\mathrm{C} 14^{\mathrm{i}}$ & $1.385(5)$ \\
$\mathrm{N} 1-\mathrm{C} 1$ & $1.332(6)$ & $\mathrm{C} 12-\mathrm{C} 13$ & $1.397(5)$ \\
$\mathrm{N} 2-\mathrm{C} 9$ & $1.330(5)$ & $\mathrm{C} 13-\mathrm{C} 18$ & $1.421(5)$ \\
$\mathrm{N} 2-\mathrm{C} 7$ & $1.361(5)$ & $\mathrm{C} 13-\mathrm{C} 14$ & $1.429(5)$ \\
$\mathrm{N} 2-\mathrm{C} 6$ & $1.467(5)$ & $\mathrm{C} 14-\mathrm{C} 12^{\mathrm{i}}$ & $1.385(5)$ \\
$\mathrm{N} 3-\mathrm{C} 9$ & $1.316(5)$ & $\mathrm{C} 14-\mathrm{C} 15$ & $1.425(5)$ \\
$\mathrm{N} 3-\mathrm{C} 8$ & $1.370(5)$ & $\mathrm{C} 15-\mathrm{C} 16$ & $1.345(5)$ \\
$\mathrm{N} 3-\mathrm{C} 10$ & $1.461(5)$ & $\mathrm{C} 15-\mathrm{H} 15$ & 0.93 \\
$\mathrm{C} 1-\mathrm{C} 2$ & $1.370(6)$ & $\mathrm{C} 16-\mathrm{C} 17$ & $1.398(6)$ \\
$\mathrm{C} 1-\mathrm{H} 1$ & 0.93 & $\mathrm{C} 16-\mathrm{H} 16$ & 0.93 \\
$\mathrm{C} 2-\mathrm{C} 3$ & $1.366(6)$ & $\mathrm{C} 17-\mathrm{C} 18$ & $1.356(5)$ \\
$\mathrm{C} 2-\mathrm{H} 2$ & 0.93 & $\mathrm{C} 17-\mathrm{H} 17$ & 0.93 \\
$\mathrm{C} 3-\mathrm{C} 4$ & $1.377(6)$ & $\mathrm{C} 18-\mathrm{H} 18$ & 0.93
\end{tabular}




\begin{tabular}{|c|c|c|c|}
\hline $\mathrm{C} 3-\mathrm{H} 3$ & 0.93 & $\mathrm{P} 1-\mathrm{F} 4 \mathrm{~B}$ & $1.518(8)$ \\
\hline $\mathrm{C} 4-\mathrm{C} 5$ & $1.369(5)$ & $\mathrm{P} 1-\mathrm{F} 3 \mathrm{~A}$ & $1.533(9)$ \\
\hline $\mathrm{C} 4-\mathrm{H} 4$ & 0.93 & $\mathrm{P} 1-\mathrm{F} 6$ & $1.544(9)$ \\
\hline $\mathrm{C} 5-\mathrm{C} 6$ & $1.502(6)$ & $\mathrm{P} 1-\mathrm{F} 1 \mathrm{~B}$ & $1.547(8)$ \\
\hline C6-H6A & 0.97 & $\mathrm{P} 1-\mathrm{F} 6 \mathrm{~A}$ & $1.548(9)$ \\
\hline C6-H6B & 0.97 & $\mathrm{P} 1-\mathrm{F} 2 \mathrm{~A}$ & $1.554(8)$ \\
\hline $\mathrm{C} 7-\mathrm{C} 8$ & $1.335(5)$ & $\mathrm{P} 1-\mathrm{F} 5$ & $1.562(9)$ \\
\hline $\mathrm{C} 7-\mathrm{H} 7$ & 0.93 & $\mathrm{P} 1-\mathrm{F} 2$ & $1.565(9)$ \\
\hline $\mathrm{C} 8-\mathrm{H} 8$ & 0.93 & $\mathrm{P} 1-\mathrm{F} 1$ & $1.566(10)$ \\
\hline $\mathrm{C} 9-\mathrm{H} 9$ & 0.93 & $\mathrm{P} 1-\mathrm{F} 5 \mathrm{~B}$ & $1.571(8)$ \\
\hline $\mathrm{C} 10-\mathrm{C} 11$ & $1.503(5)$ & $\mathrm{P} 1-\mathrm{F} 4$ & $1.572(9)$ \\
\hline $\mathrm{C} 10-\mathrm{H} 10 \mathrm{~A}$ & 0.97 & $\mathrm{P} 1-\mathrm{F} 6 \mathrm{~B}$ & $1.579(8)$ \\
\hline $\mathrm{C} 10-\mathrm{H} 10 \mathrm{~B}$ & 0.97 & & \\
\hline $\mathrm{C} 12-\mathrm{O} 1-\mathrm{C} 11$ & $113.8(3)$ & $\mathrm{O} 1-\mathrm{C} 11-\mathrm{H} 11 \mathrm{~A}$ & 110.3 \\
\hline $\mathrm{C} 5-\mathrm{N} 1-\mathrm{C} 1$ & $116.9(4)$ & $\mathrm{C} 10-\mathrm{C} 11-\mathrm{H} 11 \mathrm{~A}$ & 110.3 \\
\hline $\mathrm{C} 9-\mathrm{N} 2-\mathrm{C} 7$ & $107.9(4)$ & $\mathrm{O} 1-\mathrm{C} 11-\mathrm{H} 11 \mathrm{~B}$ & 110.3 \\
\hline $\mathrm{C} 9-\mathrm{N} 2-\mathrm{C} 6$ & $125.3(4)$ & $\mathrm{C} 10-\mathrm{C} 11-\mathrm{H} 11 \mathrm{~B}$ & 110.3 \\
\hline $\mathrm{C} 7-\mathrm{N} 2-\mathrm{C} 6$ & $126.7(4)$ & $\mathrm{H} 11 \mathrm{~A}-\mathrm{C} 11-\mathrm{H} 11 \mathrm{~B}$ & 108.5 \\
\hline $\mathrm{C} 9-\mathrm{N} 3-\mathrm{C} 8$ & $107.9(4)$ & $\mathrm{C} 14-\mathrm{C} 12-\mathrm{O} 1$ & $118.8(3)$ \\
\hline $\mathrm{C} 9-\mathrm{N} 3-\mathrm{C} 10$ & $125.7(4)$ & $\mathrm{C} 14-\mathrm{C} 12-\mathrm{C} 13$ & $122.5(3)$ \\
\hline $\mathrm{C} 8-\mathrm{N} 3-\mathrm{C} 10$ & $126.4(4)$ & $\mathrm{O} 1-\mathrm{C} 12-\mathrm{C} 13$ & $118.6(3)$ \\
\hline $\mathrm{N} 1-\mathrm{C} 1-\mathrm{C} 2$ & $123.9(4)$ & $\mathrm{C} 12-\mathrm{C} 13-\mathrm{C} 18$ & $122.7(3)$ \\
\hline $\mathrm{N} 1-\mathrm{C} 1-\mathrm{H} 1$ & 118.0 & $\mathrm{C} 12-\mathrm{C} 13-\mathrm{C} 14$ & $118.7(3)$ \\
\hline $\mathrm{C} 2-\mathrm{C} 1-\mathrm{H} 1$ & 118.0 & $\mathrm{C} 18-\mathrm{C} 13-\mathrm{C} 14$ & $118.6(3)$ \\
\hline $\mathrm{C} 3-\mathrm{C} 2-\mathrm{C} 1$ & $118.5(5)$ & $\mathrm{C} 12^{\mathrm{i}}-\mathrm{C} 14-\mathrm{C} 15$ & $122.7(3)$ \\
\hline $\mathrm{C} 3-\mathrm{C} 2-\mathrm{H} 2$ & 120.8 & $\mathrm{C} 12^{\mathrm{i}}-\mathrm{C} 14-\mathrm{C} 13$ & $118.8(3)$ \\
\hline $\mathrm{C} 1-\mathrm{C} 2-\mathrm{H} 2$ & 120.8 & $\mathrm{C} 15-\mathrm{C} 14-\mathrm{C} 13$ & $118.5(3)$ \\
\hline $\mathrm{C} 2-\mathrm{C} 3-\mathrm{C} 4$ & $118.5(5)$ & $\mathrm{C} 16-\mathrm{C} 15-\mathrm{C} 14$ & $120.6(4)$ \\
\hline $\mathrm{C} 2-\mathrm{C} 3-\mathrm{H} 3$ & 120.8 & $\mathrm{C} 16-\mathrm{C} 15-\mathrm{H} 15$ & 119.7 \\
\hline $\mathrm{C} 4-\mathrm{C} 3-\mathrm{H} 3$ & 120.8 & $\mathrm{C} 14-\mathrm{C} 15-\mathrm{H} 15$ & 119.7 \\
\hline $\mathrm{C} 5-\mathrm{C} 4-\mathrm{C} 3$ & $119.3(4)$ & $\mathrm{C} 15-\mathrm{C} 16-\mathrm{C} 17$ & $121.0(4)$ \\
\hline $\mathrm{C} 5-\mathrm{C} 4-\mathrm{H} 4$ & 120.4 & $\mathrm{C} 15-\mathrm{C} 16-\mathrm{H} 16$ & 119.5 \\
\hline $\mathrm{C} 3-\mathrm{C} 4-\mathrm{H} 4$ & 120.4 & $\mathrm{C} 17-\mathrm{C} 16-\mathrm{H} 16$ & 119.5 \\
\hline $\mathrm{N} 1-\mathrm{C} 5-\mathrm{C} 4$ & $123.0(4)$ & $\mathrm{C} 18-\mathrm{C} 17-\mathrm{C} 16$ & $121.0(4)$ \\
\hline $\mathrm{N} 1-\mathrm{C} 5-\mathrm{C} 6$ & $115.1(4)$ & $\mathrm{C} 18-\mathrm{C} 17-\mathrm{H} 17$ & 119.5 \\
\hline $\mathrm{C} 4-\mathrm{C} 5-\mathrm{C} 6$ & $121.9(4)$ & $\mathrm{C} 16-\mathrm{C} 17-\mathrm{H} 17$ & 119.5 \\
\hline $\mathrm{N} 2-\mathrm{C} 6-\mathrm{C} 5$ & $112.2(3)$ & $\mathrm{C} 17-\mathrm{C} 18-\mathrm{C} 13$ & $120.3(4)$ \\
\hline $\mathrm{N} 2-\mathrm{C} 6-\mathrm{H} 6 \mathrm{~A}$ & 109.2 & $\mathrm{C} 17-\mathrm{C} 18-\mathrm{H} 18$ & 119.9 \\
\hline $\mathrm{C} 5-\mathrm{C} 6-\mathrm{H} 6 \mathrm{~A}$ & 109.2 & $\mathrm{C} 13-\mathrm{C} 18-\mathrm{H} 18$ & 119.9 \\
\hline $\mathrm{N} 2-\mathrm{C} 6-\mathrm{H} 6 \mathrm{~B}$ & 109.2 & $\mathrm{~F} 4 \mathrm{~B}-\mathrm{P} 1-\mathrm{F} 1 \mathrm{~B}$ & $94.3(8)$ \\
\hline $\mathrm{C} 5-\mathrm{C} 6-\mathrm{H} 6 \mathrm{~B}$ & 109.2 & $\mathrm{~F} 3 \mathrm{~A}-\mathrm{P} 1-\mathrm{F} 6 \mathrm{~A}$ & $96.6(10)$ \\
\hline $\mathrm{H} 6 \mathrm{~A}-\mathrm{C} 6-\mathrm{H} 6 \mathrm{~B}$ & 107.9 & $\mathrm{~F} 3 \mathrm{~A}-\mathrm{P} 1-\mathrm{F} 2 \mathrm{~A}$ & $97.0(10)$ \\
\hline $\mathrm{C} 8-\mathrm{C} 7-\mathrm{N} 2$ & $107.5(4)$ & $\mathrm{F} 6 \mathrm{~A}-\mathrm{P} 1-\mathrm{F} 2 \mathrm{~A}$ & $92.4(9)$ \\
\hline $\mathrm{C} 8-\mathrm{C} 7-\mathrm{H} 7$ & 126.3 & $\mathrm{~F} 6-\mathrm{P} 1-\mathrm{F} 5$ & $87.0(10)$ \\
\hline $\mathrm{N} 2-\mathrm{C} 7-\mathrm{H} 7$ & 126.3 & $\mathrm{~F} 6-\mathrm{P} 1-\mathrm{F} 2$ & $83.7(10)$ \\
\hline $\mathrm{C} 7-\mathrm{C} 8-\mathrm{N} 3$ & $107.5(4)$ & $\mathrm{F} 5-\mathrm{P} 1-\mathrm{F} 2$ & $87.4(10)$ \\
\hline $\mathrm{C} 7-\mathrm{C} 8-\mathrm{H} 8$ & 126.2 & $\mathrm{~F} 6-\mathrm{P} 1-\mathrm{F} 1$ & $156.8(13)$ \\
\hline
\end{tabular}




\begin{tabular}{|c|c|c|c|}
\hline $\mathrm{N} 3-\mathrm{C} 8-\mathrm{H} 8$ & 126.2 & $\mathrm{~F} 5-\mathrm{P} 1-\mathrm{F} 1$ & $77.1(11)$ \\
\hline $\mathrm{N} 3-\mathrm{C} 9-\mathrm{N} 2$ & $109.1(4)$ & $\mathrm{F} 2-\mathrm{P} 1-\mathrm{F} 1$ & $78.9(12)$ \\
\hline $\mathrm{N} 3-\mathrm{C} 9-\mathrm{H} 9$ & 125.4 & $\mathrm{~F} 4 \mathrm{~B}-\mathrm{P} 1-\mathrm{F} 5 \mathrm{~B}$ & $93.5(8)$ \\
\hline $\mathrm{N} 2-\mathrm{C} 9-\mathrm{H} 9$ & 125.4 & $\mathrm{~F} 1 \mathrm{~B}-\mathrm{P} 1-\mathrm{F} 5 \mathrm{~B}$ & $118.9(9)$ \\
\hline $\mathrm{N} 3-\mathrm{C} 10-\mathrm{C} 11$ & $112.8(3)$ & $\mathrm{F} 6-\mathrm{P} 1-\mathrm{F} 4$ & $91.2(10)$ \\
\hline $\mathrm{N} 3-\mathrm{C} 10-\mathrm{H} 10 \mathrm{~A}$ & 109.0 & $\mathrm{~F} 5-\mathrm{P} 1-\mathrm{F} 4$ & $97.5(10)$ \\
\hline $\mathrm{C} 11-\mathrm{C} 10-\mathrm{H} 10 \mathrm{~A}$ & 109.0 & $\mathrm{~F} 2-\mathrm{P} 1-\mathrm{F} 4$ & $172.8(9)$ \\
\hline N3-C10-H10B & 109.0 & $\mathrm{~F} 1-\mathrm{P} 1-\mathrm{F} 4$ & $107.3(11)$ \\
\hline $\mathrm{C} 11-\mathrm{C} 10-\mathrm{H} 10 \mathrm{~B}$ & 109.0 & $\mathrm{~F} 4 \mathrm{~B}-\mathrm{P} 1-\mathrm{F} 6 \mathrm{~B}$ & $84.2(7)$ \\
\hline $\mathrm{H} 10 \mathrm{~A}-\mathrm{C} 10-\mathrm{H} 10 \mathrm{~B}$ & 107.8 & $\mathrm{~F} 1 \mathrm{~B}-\mathrm{P} 1-\mathrm{F} 6 \mathrm{~B}$ & $161.1(9)$ \\
\hline $\mathrm{O} 1-\mathrm{C} 11-\mathrm{C} 10$ & $107.3(3)$ & $\mathrm{F} 5 \mathrm{~B}-\mathrm{P} 1-\mathrm{F} 6 \mathrm{~B}$ & $79.9(7)$ \\
\hline $\mathrm{C} 5-\mathrm{N} 1-\mathrm{C} 1-\mathrm{C} 2$ & $0.5(7)$ & $\mathrm{C} 9-\mathrm{N} 3-\mathrm{C} 10-\mathrm{C} 11$ & $83.6(5)$ \\
\hline $\mathrm{N} 1-\mathrm{C} 1-\mathrm{C} 2-\mathrm{C} 3$ & $0.6(8)$ & $\mathrm{C} 8-\mathrm{N} 3-\mathrm{C} 10-\mathrm{C} 11$ & $-98.5(4)$ \\
\hline $\mathrm{C} 1-\mathrm{C} 2-\mathrm{C} 3-\mathrm{C} 4$ & $-0.4(7)$ & $\mathrm{C} 12-\mathrm{O} 1-\mathrm{C} 11-\mathrm{C} 10$ & $-174.0(3)$ \\
\hline $\mathrm{C} 2-\mathrm{C} 3-\mathrm{C} 4-\mathrm{C} 5$ & $-0.8(6)$ & $\mathrm{N} 3-\mathrm{C} 10-\mathrm{C} 11-\mathrm{O} 1$ & $-70.3(4)$ \\
\hline $\mathrm{C} 1-\mathrm{N} 1-\mathrm{C} 5-\mathrm{C} 4$ & $-1.9(7)$ & $\mathrm{C} 11-\mathrm{O} 1-\mathrm{C} 12-\mathrm{C} 14^{\mathrm{i}}$ & $-96.3(4)$ \\
\hline $\mathrm{C} 1-\mathrm{N} 1-\mathrm{C} 5-\mathrm{C} 6$ & $176.5(4)$ & $\mathrm{C} 11-\mathrm{O} 1-\mathrm{C} 12-\mathrm{C} 13$ & $87.7(4)$ \\
\hline $\mathrm{C} 3-\mathrm{C} 4-\mathrm{C} 5-\mathrm{N} 1$ & $2.1(7)$ & $\mathrm{C} 14-\mathrm{C} 12-\mathrm{C} 13-\mathrm{C} 18$ & $-179.0(3)$ \\
\hline $\mathrm{C} 3-\mathrm{C} 4-\mathrm{C} 5-\mathrm{C} 6$ & $-176.2(4)$ & $\mathrm{O} 1-\mathrm{C} 12-\mathrm{C} 13-\mathrm{C} 18$ & $-3.1(5)$ \\
\hline $\mathrm{C} 9-\mathrm{N} 2-\mathrm{C} 6-\mathrm{C} 5$ & $-93.4(5)$ & $\mathrm{C} 14-\mathrm{C} 12-\mathrm{C} 13-\mathrm{C} 14$ & $1.5(6)$ \\
\hline $\mathrm{C} 7-\mathrm{N} 2-\mathrm{C} 6-\mathrm{C} 5$ & $84.1(5)$ & $\mathrm{O} 1-\mathrm{C} 12-\mathrm{C} 13-\mathrm{C} 14$ & $177.3(3)$ \\
\hline $\mathrm{N} 1-\mathrm{C} 5-\mathrm{C} 6-\mathrm{N} 2$ & $55.0(5)$ & $\mathrm{C} 12-\mathrm{C} 13-\mathrm{C} 14-\mathrm{C} 12^{\mathrm{i}}$ & $-1.4(6)$ \\
\hline $\mathrm{C} 4-\mathrm{C} 5-\mathrm{C} 6-\mathrm{N} 2$ & $-126.6(4)$ & $\mathrm{C} 18-\mathrm{C} 13-\mathrm{C} 14-\mathrm{C} 12^{\mathrm{i}}$ & $179.0(3)$ \\
\hline $\mathrm{C} 9-\mathrm{N} 2-\mathrm{C} 7-\mathrm{C} 8$ & $-0.5(5)$ & $\mathrm{C} 12-\mathrm{C} 13-\mathrm{C} 14-\mathrm{C} 15$ & $178.7(3)$ \\
\hline $\mathrm{C} 6-\mathrm{N} 2-\mathrm{C} 7-\mathrm{C} 8$ & $-178.3(4)$ & $\mathrm{C} 18-\mathrm{C} 13-\mathrm{C} 14-\mathrm{C} 15$ & $-0.8(5)$ \\
\hline $\mathrm{N} 2-\mathrm{C} 7-\mathrm{C} 8-\mathrm{N} 3$ & $0.3(5)$ & $\mathrm{C} 12 \mathrm{i}-\mathrm{C} 14-\mathrm{C} 15-\mathrm{C} 16$ & $-180.0(4)$ \\
\hline $\mathrm{C} 9-\mathrm{N} 3-\mathrm{C} 8-\mathrm{C} 7$ & $0.1(4)$ & $\mathrm{C} 13-\mathrm{C} 14-\mathrm{C} 15-\mathrm{C} 16$ & $-0.1(6)$ \\
\hline $\mathrm{C} 10-\mathrm{N} 3-\mathrm{C} 8-\mathrm{C} 7$ & $-178.1(3)$ & $\mathrm{C} 14-\mathrm{C} 15-\mathrm{C} 16-\mathrm{C} 17$ & $1.3(7)$ \\
\hline $\mathrm{C} 8-\mathrm{N} 3-\mathrm{C} 9-\mathrm{N} 2$ & $-0.4(4)$ & $\mathrm{C} 15-\mathrm{C} 16-\mathrm{C} 17-\mathrm{C} 18$ & $-1.5(7)$ \\
\hline $\mathrm{C} 10-\mathrm{N} 3-\mathrm{C} 9-\mathrm{N} 2$ & $177.8(3)$ & $\mathrm{C} 16-\mathrm{C} 17-\mathrm{C} 18-\mathrm{C} 13$ & $0.5(7)$ \\
\hline $\mathrm{C} 7-\mathrm{N} 2-\mathrm{C} 9-\mathrm{N} 3$ & $0.5(4)$ & $\mathrm{C} 12-\mathrm{C} 13-\mathrm{C} 18-\mathrm{C} 17$ & $-178.9(4)$ \\
\hline $\mathrm{C} 6-\mathrm{N} 2-\mathrm{C} 9-\mathrm{N} 3$ & $178.4(3)$ & $\mathrm{C} 14-\mathrm{C} 13-\mathrm{C} 18-\mathrm{C} 17$ & $0.6(6)$ \\
\hline
\end{tabular}

Symmetry code: (i) $-x+1,-y+1,-z+2$. 